\title{
High-Flow Nasal Cannula Oxygen in Patients with Acute Respiratory Failure and Do-Not-Intubate or Do-Not-Resuscitate Orders: A Systematic Review
}

\author{
Michael E Wilson, MD',2,3*, Aniket Mittal, MBBS', Claudia C Dobler, MD, PhD', \\ J Randall Curtis, MD, MPH'5,6, Abdul M Majzoub, MD4, Jalal Soleimani, MD1', Ognjen Gajic, MD,MSc', \\ Patricia J Erwin 7 , Victor M Montori, MD, MSc ${ }^{3,8}$, M Hassan Murad, MD, MPH',4
}

\begin{abstract}
${ }^{1}$ Division of Pulmonary and Critical Care Medicine, Mayo Clinic, Rochester, Minnesota; ${ }^{2}$ Robert D. and Patricia E. Kern Center for the Science of Health Care Delivery, Mayo Clinic, Rochester, Minnesota; ${ }^{3}$ Knowledge and Evaluation Research Unit, Mayo Clinic, Rochester, Minnesota; ${ }^{4}$ Evidence-Based Practice Center, Mayo Clinic, Rochester, Minnesota; ${ }^{5}$ Division of Pulmonary, Critical Care, and Sleep Medicine, Harborview Medical Center, University of Washington, Seattle, Washington; ${ }^{6}$ Cambia Palliative Care Center of Excellence, University of Washington, Seattle, Washington; ${ }^{7}$ Medical Library, Mayo Clinic, Rochester, Minnesota; ${ }^{8}$ Department of Medicine, Mayo Clinic, Rochester, Minnesota.
\end{abstract}

BACKGROUND AND OBJECTIVES: High-flow nasal cannula (HFNC) oxygen may provide tailored benefits in patients with preset treatment limitations. The objective of this study was to assess the effectiveness of HFNC oxygen in patients with do-not-intubate (DNI) and/or do-notresuscitate (DNR) orders.

METHODS: We conducted a systematic review of interventional and observational studies. A search was performed using MEDLINE, EMBASE, CINAHL, Scopus, and Web of Science, from inception to October 15, 2018.

RESULTS: We included six studies evaluating 293 patients. All studies had a high risk of bias. The hospital mortality rates of patients with $\mathrm{DNI}$ and/or DNR orders receiving HFNC oxygen were variable and ranged from $40 \%$ to $87 \%$. In two before and after studies, the initiation of HFNC oxygen was associated with improved oxygenation and reduced respiratory rates. One comparative study found no difference in dyspnea reduction or morphine doses between patients using HFNC oxygen versus conventional oxygen. No studies evaluated quality of life in survivors or quality of death in nonsurvivors. HFNC was generally well tolerated with few adverse events identified.

CONCLUSIONS: While HFNC oxygen remains a viable treatment option for hospitalized patients who have acute respiratory failure and a DNI and/or DNR order, there is a paucity of high-quality, comparative, effectiveness data to guide the usage of HFNC oxygen compared with other treatments, such as noninvasive ventilation, conventional oxygen, and palliative opioids. Journal of Hospital Medicine 2020;15:101-106. () 2020 Society of Hospital Medicine

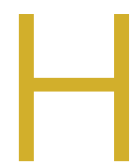
igh-flow nasal cannula (HFNC) oxygen therapy is effective in treating adults with acute hypoxemic respiratory failure, and to a lesser extent acute hypercapnic respiratory failure. ${ }^{1-3} \mathrm{HFNC}$ oxygen is capable of delivering oxygen with flows of 30-60 liters/minute, and can provide a high fraction of inspired oxygen, flush anatomic dead space, augment respiratory efforts, and provide mild continuous positive airway pressure effects. Several systematic reviews and meta-analyses have evaluated the effectiveness of HFNC oxygen and have shown modestly lower rates of intubation compared with conventional oxygen ${ }^{4,5}$ and similar intubation rates compared with noninvasive positive pressure ventilation. ${ }^{4-9}$ Although one randomized trial showed a lower risk of 90-day mortality for HFNC oxygen compared with either conventional oxygen

Corresponding Author: Michael E. Wilson, MD; Email: wilson.michael1 @mayo.edu

Published online first November 20, 2019.

Find Additional Supporting Information in the online version of this article.

Received: July 9, 2019; Revised: September 9, 2019;

Accepted: September 16, 2019

() 2020 Society of Hospital Medicine DOI 10.12788/jhm.3329 or noninvasive positive pressure ventilation, several meta-analyses have shown no difference in intensive care unit (ICU) mortality. ${ }^{4,6,8,10}$ The majority of studies have shown improvements in oxygenation, comfort, dyspnea scores, and breathing pattern with the initiation of HFNC oxygen. ${ }^{6}$

While the evidence to support the use of HFNC oxygen in patients with nonhypercapnic acute hypoxemic respiratory failure is growing, this evidence is based on patients enrolled in clinical trials who have no treatment limitations and consent to intubation if necessary. Indeed, several, if not all, randomized trials evaluating HFNC oxygen excluded patients who had do-not-intubate (DNI) or do-not-resuscitate (DNR) orders. 1,2,11 For patients with acute respiratory failure whose primary goal is not to extend life or utilize life support interventions such as invasive mechanical ventilation, HFNC oxygen may offer several benefits compared with other treatment options such as noninvasive positive pressure ventilation, conventional oxygen therapy, or palliative opioid therapy (Appendix Table 1). Determining which treatment options to use depends on the goals of care of the individual patient and the reasonable ability of a particular treatment to help the patient achieve those goals.

While a recent systematic review evaluated the existing evidence regarding the utility and outcomes of noninvasive positive 
pressure ventilation in adult patients with DNI orders, ${ }^{12}$ a systematic review evaluating the evidence and rationale for HFNC oxygen in patients with DNI and/or DNR orders is lacking. Assessing such evidence is necessary to help clinicians and patients determine appropriate treatment choices and establish research priorities. Therefore, our primary objective was to determine what were the following outcomes: mortality, dyspnea, work of breathing, opioid doses, and quality of life in patients who received HFNC oxygen for acute respiratory failure and had a DNI and/or DNR order.

\section{METHODS}

We conducted a systematic review of studies that evaluated patients who used HFNC oxygen for acute respiratory failure and had a DNI and/or DNR order. We reported the results using the Preferred Reporting Items for Systematic Reviews and Meta-Analyses (PRISMA) statements. ${ }^{13}$ This review was registered with the PROSPERO registry, CRD42017059914.

We included studies that enrolled patients who were (1) hospitalized, (2) >18 years old, (3) had an acute respiratory failure of any cause, (4) received HFNC oxygen, and (5) had a DNI or DNR or comfort measures only order. We included publications of all study designs (interventional, observational, and posthoc analyses) and all languages. We excluded studies that enrolled $<5$ patients. If necessary, we contacted the authors of the included studies for additional information.

Our search strategy included the following databases from inception to October 14, 2018: PubMed, MEDLINE, CINAHL, MICROMEDEX, EMBASE, Web of Science, and Scopus. The database-specific search strategy was developed using an experienced librarian (Appendix Table 2). In addition, we screened the reference lists of systematic reviews as well as the included studies to find additional relevant articles. Two authors (AM, MEW) independently assessed the inclusion criteria of the titles and abstracts that were identified in the search. In addition, these two authors abstracted relevant data of the included studies.

The primary outcomes were mortality, dyspnea and work of breathing, quality of life, and reduction of opioid doses. Secondary, posthoc, outcomes included the transition to noninvasive positive pressure ventilation (NPPV), tolerance of HFNC, adverse events, and quality of death in nonsurvivors. The risk of bias was evaluated using a modified Newcastle-Ottawa Quality Assessment Scale (Appendix Table 3).

\section{RESULTS}

Using the search strategy, we identified 2,757 citations and included 301 of these in the full-text review (Figure). We included six studies, which enrolled 293 patients in the final systematic review. Table 1 summarizes the characteristics of the included investigations, all of which were observational studies. ${ }^{15-20}$ The studies were conducted in the United States of America $(n=3)$, Europe $(n=2)$, and Asia $(n=1)$. Two studies were conducted in the general ICU populations and included patients with hypoxemic respiratory failure only. Four studies were conducted in cancer populations in the hospital wards or ICU and did not specify the type of respiratory failure (hypoxemic versus hypercapnic). Two studies included patients with DNI orders only. ${ }^{15,20}$
One study included patients with DNR orders only (DNI orders were excluded). ${ }^{17}$ Three studies included patients with both DNR and DNI orders. ${ }^{16,18,19}$ The numbers of enrolled patients with treatment limitations were generally low, with the two largest studies including 101 patients each on HFNC oxygen. ${ }^{18,19}$

\section{Risk of Bias}

All included studies had a high risk of bias (Table 2). A high risk of bias was suggested because the investigations were single-center studies with unclear patient selection methods, did not explicitly report how decisions to limit treatments were made, and did not explicitly differentiate and separately analyze patients with "comfort measures only" goals of care.

\section{Mortality}

The hospital mortality rates of patients with DNI and/or DNR orders receiving HFNC were variable and ranged from $40 \%$ to $87 \%$. In the two studies enrolling general ICU patient populations, the hospital mortality rates ranged from $40 \%$ to $60 \%$. In the four studies enrolling patients with active malignancy, the hospital mortality rates ranged from $75 \%$ to $87 \%$. No studies compared mortality rates with and without DNI and/or DNR orders.

\section{Dyspnea, Work of Breathing, and Reduction in Opi- oid Doses}

The impact of HFNC oxygen on symptom relief was reported in one retrospective observational study (published as a conference abstract only to date), which compared the effect of HFNC oxygen $(n=101)$ with conventional oxygen $(n=110) .{ }^{18}$ At first evaluation after hospital admission to a palliative care unit (after the patients had previously been started on either conventional oxygen or high-flow oxygen), patients in the HFNC oxygen group had worse (higher) dyspnea scores compared with patients who used conventional oxygen (Edmonton Symptom Assessment Scale score of 7.5 versus 5, $P<.001$ ). At follow-up, approximately 24 hours after admission to the hospital palliative care unit, there was no difference in the change of dyspnea between the HFNC oxygen group (dyspnea score change of 0 ) and the conventional oxygen group (dyspnea score change of $-1, P=.18$. In the same study, there was also no significant difference in the morphine dose requirement in each group, and exact doses were not reported.

Two studies reported improvement in oxygen saturation and respiratory rate after HFNC oxygen initiation (compared with before HFNC initiation). ${ }^{16,20}$ Oxygen saturation increased from $89 \%$ to $95 \%, P<.01$, in one study and $92 \%$ to $97 \%, P<.01$, in a second study. The respiratory rate decreased from 31 to 25 breaths/minute in one study, and from 28 to 25 breaths/minute in a second study (both $P<.01$ ).

\section{Quality of Life}

No studies evaluated the quality of life of survivors.

\section{Secondary Outcomes}

Transition to Noninvasive Positive Pressure Ventilation

The proportion of patients who transitioned from HFNC oxy- 
TABLE 1. Characteristics of Studies of High-Flow Nasal Cannula in Patients with Do-Not-Intubate or Do-Not-Resuscitate Orders

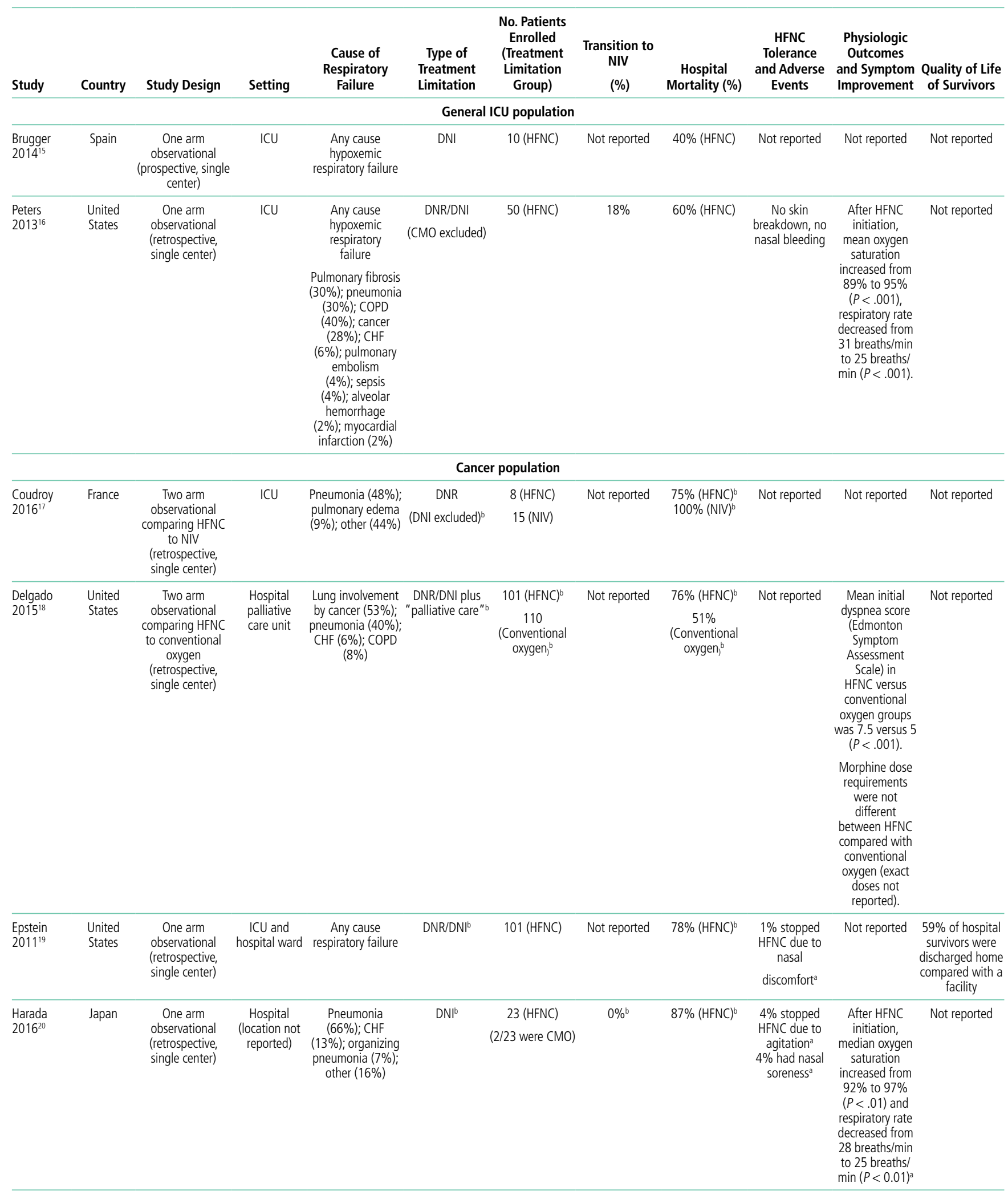

aalues are for DNI or DNR orders and full-code cohort combined.

bValues were obtained via author communication.

Abbreviations: $\mathrm{CHF}$, congestive heart failure; $\mathrm{CMO}$, comfort measures only; COPD, chronic obstructive pulmonary disease; DNI, do not intubate; DNR, do not resuscitate; HFNC, high-flow nasal cannula; ICU, intensive care unit; NIV, noninvasive ventilation. 
TABLE 2. Risk of Bias

\begin{tabular}{|c|c|c|c|c|c|c|c|}
\hline Study & Sample Representativeness & Sample Size & Nonexposed Cohort & $\begin{array}{c}\text { Ascertainment of } \\
\text { Exposure No. } 1\end{array}$ & $\begin{array}{l}\text { Ascertainment of } \\
\text { Exposure No. } 2\end{array}$ & Total Score & Risk of Bias \\
\hline Brugger $2014^{15}$ & 0 & 0 & 0 & 0 & 0 & 0 & High \\
\hline Peters $2013^{16}$ & 0 & 1 & 0 & 0 & 1 & 2 & High \\
\hline Cuodroy $2016^{17}$ & 0 & 0 & 0 & 0 & 0 & 0 & High \\
\hline Delgado $2015^{18}$ & 0 & 1 & 0 & 0 & 0 & 1 & High \\
\hline Epstein $2011^{19}$ & 0 & 1 & 1 & 0 & 0 & 2 & High \\
\hline Harada $2016^{20}$ & 0 & 0 & 1 & 0 & 1 & 2 & High \\
\hline
\end{tabular}

Scoring: low risk of bias: total score, 3-5 points; high risk of bias: total score, 0-2 points

gen to NPPV was relatively low in the two studies that reported this outcome, ranging from $0 \%{ }^{20}$ to $18 \% .^{16}$ In one observational study of a general ICU population, 9/50 (18\%) of patients transitioned from HFNC oxygen to NPPV. There was no statistically significant difference in hospital mortality rates among those who progressed to NPPV (67\%) versus those who did not progress to NPPV (58\%), $P=.72$.

\section{Tolerance of HFNC and Adverse Events}

HFNC oxygen was generally well tolerated based on the assessment of three studies (Table 1). One study reported no adverse events, ${ }^{16}$ one study reported that HFNC oxygen had to be discontinued because of nasal discomfort in $1 \%$ of patients, ${ }^{19}$ and a second study reported that HFNC oxygen had to be discontinued because of agitation in $4 \%$ of patients. ${ }^{20}$

\section{Quality of Death in Nonsurvivors}

No studies evaluated the quality of death in those patients who died.

\section{DISCUSSION}

In this systematic review of six studies, all with a high risk of bias, a significant proportion of patients with a DNI and/or DNR order who used HFNC oxygen survived to hospital discharge. Oxygen saturation and respiratory rate consistently improved in the three studies that reported these outcomes. Only one study (published as a conference abstract only to date), ${ }^{18}$ however, measured patient-important outcomes related to symptom management and found no significant difference in dyspnea or morphine dose requirements in patients on HFNC oxygen compared with patients on conventional oxygen. HFNC oxygen was generally well tolerated and only had to be stopped in $<5 \%$ of patients due to intolerance. We found no studies that assessed the quality of life in survivors or the quality of death in nonsurvivors.

Based on the limited evidence in the included studies, HFNC may be a viable treatment option for patients with preset treatment limitations who have acute respiratory failurewith potential benefits of improved oxygenation, decreased respiratory rates, and hospital survival in a proportion of pa- tients. Nevertheless, this systematic review highlights the vast paucity of data available to guide the use of HFNC oxygen in patients with treatment limitations and acute respiratory failure. Only a few studies, which were at high risk of bias, have been conducted on this topic to date. There is an inadequate evidence base to evaluate the comparative effectiveness of HFNC oxygen (versus NPPV versus conventional oxygen versus palliative opioids) in patients with $\mathrm{DNI}$ orders or comfort measures only orders.

Our review included two studies that evaluated the comparative effectiveness of HFNC oxygen in patients with DNI and/ or DNR orders. The first retrospective observational study compared HFNC oxygen with conventional oxygen in patients with DNR and DNI orders and malignancy - and found no change in dyspnea-but did note an increase in mortality with HFNC oxygen (76\% versus $51 \%) .{ }^{18}$ The second observational study compared HFNC oxygen with NPPV in patients with DNR orders with malignancy noted no difference in mortality. ${ }^{17}$ In patients with full-code orders, systematic reviews have shown that HFNC oxygen (compared with conventional oxygen) was associated with possible reductions in intubation rates, respiratory rates, and improvements in oxygenation - with no difference in mortality, dyspnea, patient comfort, or ICU/hospital length of stay. Compared with NPPV, HFNC oxygen was associated with similar rates of intubation and mortality. ${ }^{4-6,21}$

Future studies in patients with acute respiratory failure and DNI and/or DNR orders should identify which treatment modality (HFNC oxygen compared with other modalities, such as NPPV, conventional oxygen, with or without palliative opioids) impacts outcomes, such as dyspnea reduction while maintaining an alert mental status, short- and long-term quality of life in survivors, and quality of death in nonsurvivors. Future studies should also identify the optimal treatment pathway to utilize when patients using HFNC oxygen fail this therapy (eg, transition to NPPV versus intensifying palliative opioids) as well as the optimal process to withdraw palliative HFNC oxygen. ${ }^{22}$ Identifying which patient populations may benefit from different treatment pathways should also be considered as different treatment strategies may be more beneficial in different patient populations (eg, based on cause and sever- 


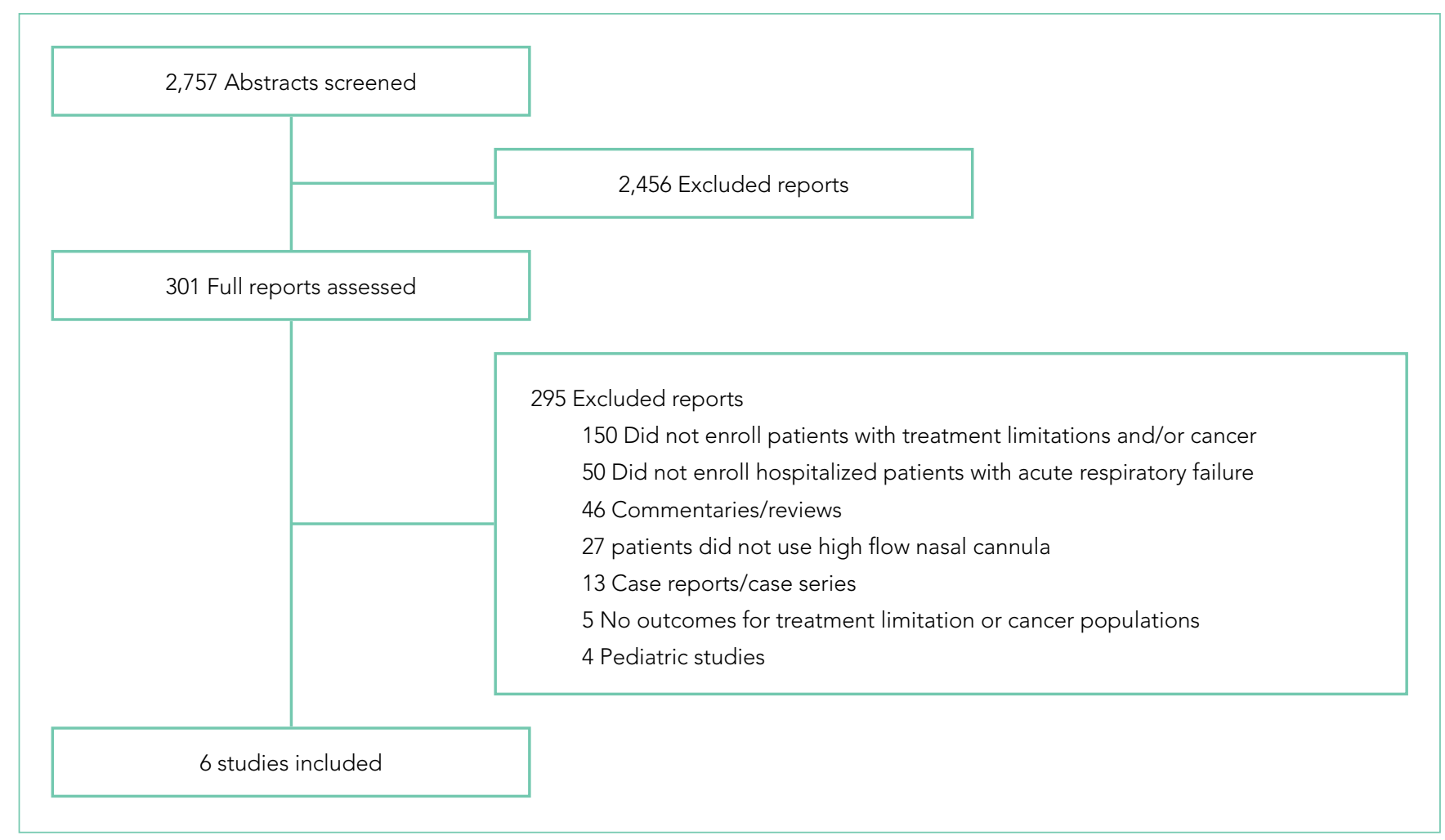

FIG. Search Strategy

ity of acute respiratory failure). In addition, it should be noted that the primary goal of care might affect which outcomes are the most important to measure. While patients with comfort measures only, orders usually have a primary goal to prepare for a high-quality death, patients with DNI and/or DNR orders (but without comfort measures only orders) may have a primary goal to survive-but with the desire not to endure the high burden of intubation and mechanical ventilation if it became necessary. Finally, future studies should utilize high-quality study designs (eg, randomized controlled trials) that enable robust evaluation of comparative effectiveness of clinically relevant treatment strategies.

While several previous systematic reviews have evaluated the efficacy of HFNC in patients with acute respiratory failure without preset limitations on life support; to our knowledge, this is the first systematic review to assess outcomes in patients rigorously with preset treatment limitations. Our review is, however, limited by the high risk of bias of the studies that were included (single-center nature, retrospective observational study designs, small sample sizes, and lack of a description of how DNI and/or DNR statuses were determined) as well as the small number of studies available to be included.

\section{CONCLUSIONS}

This systematic review points to a significant evidence gap in our understanding of the role for HFNC oxygen (compared with other acceptable alternative treatment strategies) in adult patients with acute respiratory failure who have DNI and/or DNR orders. Further high-quality research is needed to ex- plore these unanswered questions in an effort to best treat, guide, and engage in optimal end-of-life decision making among patients with acute respiratory failure.

Disclosures: The authors report no conflicts of interest

\section{References}

1. Frat J-P, Thille AW, Mercat A, et al. High-flow oxygen through nasal cannula in acute hypoxemic respiratory failure. N Eng J Med. 2015;372(23):2185-2196. https://doi.org/ 10.1056/NEJMoa1503326.

2. Stephan $F$, Barrucand $B$, Petit $P$, et al. High-flow nasal oxygen vs noninvasive positive airway pressure in hypoxemic patients after cardiothoracic surgery: a randomized clinical trial. JAMA. 2015;313(23):2331-2339. https://doi.org/ 10.1001/jama.2015.5213.

3. Lee MK, Choi J, Park B, et al. High flow nasal cannulae oxygen therapy in acute-moderate hypercapnic respiratory failure. Clin Respir J. 2018;12(6):2046-2056. https://doi.org/10.1111/crj.12772 28.

4. Ni YN, Luo J, Yu H, et al. Can high-flow nasal cannula reduce the rate of endotracheal intubation in adult patients with acute respiratory failure compared with conventional oxygen therapy and noninvasive positive pressure ventilation?: a systematic review and meta-analysis. Chest. 2017;151(4):764775. https://doi.org/10.1016/j.chest.2017.01.004.

5. Ou X, Hua Y, Liu J, Gong C, Zhao W. Effect of high-flow nasal cannula oxygen therapy in adults with acute hypoxemic respiratory failure: a meta-analysis of randomized controlled trials. CMAJ. 2017;189(7):E260-E267. https://doi. org/10.1503/cmaj.160570.

6. Monro-Somerville T, Sim M, Ruddy J, Vilas M, Gillies MA. The effect of highflow nasal cannula oxygen therapy on mortality and intubation rate in acute respiratory failure: a systematic review and meta-analysis. Crit Care Med. 2017;45(4):e449-e456. https://doi.org/10.1097/CCM.0000000000002091.

7. Maitra S, Som A, Bhattacharjee S, Arora MK, Baidya DK. Comparison of highflow nasal oxygen therapy with conventional oxygen therapy and noninvasive ventilation in adult patients with acute hypoxemic respiratory failure: a meta-analysis and systematic review. J Crit Care. 2016;35:138-144. https:// doi.org/10.1016/j.jcrc.2016.05.013 
8. Nedel WL, Deutschendorf C, Moraes Rodrigues Filho E. High-flow nasal cannula in critically ill subjects with or at risk for respiratory failure: a systematic review and meta-analysis. Respir Care. 2017;62(1):123-132. https://doi. org/10.4187/respcare.04831.

9. Zhu $Y$, Yin $H$, Zhang R, Wei J. High-flow nasal cannula oxygen therapy vs conventional oxygen therapy in cardiac surgical patients: a meta-analysis. $J$ Crit Care. 2017;38:123-128. https://doi.org/10.1016/j.jcrc.2016.10.027.

10. Leeies M, Flynn $E$, Turgeon AF, et al. High-flow oxygen via nasal cannulae in patients with acute hypoxemic respiratory failure: a systematic review and meta-analysis. Syst Rev. 2017;6(1):202. https://doi.org/10.1186/s13643-0170593-5.

11. Hernandez G, Vaquero C, Gonzalez P, et al. Effect of postextubation highflow nasal cannula vs conventional oxygen therapy on reintubation in low-risk patients: a randomized clinical trial. JAMA. 2016;315(13):1354-1361. https:// doi.org/10.1001/jama.2016.2711.

12. Wilson ME, Majzoub AM, Dobler CC, et al. Noninvasive ventilation in patients with do-not-intubate and comfort-measures-only orders: a systematic review and meta-analysis. Crit Care Med. 2018. 46(8):1209-1216. https://doi. org/10.1097/CCM.0000000000003082.

13. Moher D, Liberati A, Tetzlaff J, Altman DG. Preferred reporting items for systematic reviews and meta-analyses: the PRISMA statement. BMJ. 2009;339:b2535. https://doi.org/10.1136/bmj.b2535

14. Stroup DF, Berlin JA, Morton SC, et al. Meta-analysis of observational studies in epidemiology: a proposal for reporting. Meta-analysis Of Observational Studies in Epidemiology (MOOSE) group. JAMA. 2000;283(15):2008-2012. https://doi.org/10.1001/jama.283.15.2008

15. Brugger SC, Rodriguez S, Domingo J, et al. High-flow nasal cannula therapy
(HFNC) for patients with severe acute respiratory failure and do not intubate orders. Pilot study. Palliative Medicine. 2014;28(6):755

16. Peters SG, Holets SR, Gay PC. High-flow nasal cannula therapy in do-not-intubate patients with hypoxemic respiratory distress. Respir Care. 2013;58(4):597-600. https://doi.org/10.4187/respcare.01887.

17. Coudroy R, Jamet A, Petua P, Robert R, Frat JP, Thille AW. High-flow nasal cannula oxygen therapy versus noninvasive ventilation in immunocompromised patients with acute respiratory failure: an observational cohort study. Ann Intensive Care. 2016;6(1):45. https://doi.org/10.1186/s13613-016-0151-7.

18. Delgado-Guay MO, Rodriguez-Nunez A, Adegboyega $O O$, et al. Characteristics and outcomes of advanced cancer patients admitted to an acute palliative care unit (PCU) with severe dyspnea receiving high flow oxygen (HFO). Journal of Clinical Oncology Conference. 2015;33(29 SUPPL. 1):247.

19. Epstein AS, Hartridge-Lambert SK, Ramaker JS, Voigt LP, Portlock CS. Humidified high-flow nasal oxygen utilization in patients with cancer at Memorial Sloan-Kettering Cancer Center. J Palliat Med. 2011;14(7):835-839. https:// doi.org/10.1089/jpm.2011.0005.

20. Harada K, Kurosawa S, Hino Y, et al. Clinical utility of high-flow nasal cannula oxygen therapy for acute respiratory failure in patients with hematological disease. Springerplus. 2016;5(1):512. https://doi.org/10.1186/s40064-016-2161-1.

21. Rochwerg B, Granton D, Wang DX, et al. High flow nasal cannula compared with conventional oxygen therapy for acute hypoxemic respiratory failure: a systematic review and meta-analysis. Intensive Care Med. 2019;45(5):563572. https://doi.org/10.1007/s00134-019-05590-5.

22. Halpern SD, Hansen-Flaschen J. Terminal withdrawal of life-sustaining supplemental oxygen. JAMA. 2006;296(11):1397-1400. https://doi.org/10.1001/ jama.296.11.1397. 